\section{Synchronizing Nuclear Import of Ribosomal Proteins with Ribosome Assembly}

\author{
Dieter Kressler, ${ }^{1,2 *} \dagger$ Gert Bange, ${ }^{1 *}$ Yutaka Ogawa, ${ }^{3}$ Goran Stjepanovic, ${ }^{1}$ Bettina Bradatsch, ${ }^{1}$ \\ Dagmar Pratte, ${ }^{2}$ Stefan Amlacher, ${ }^{1}$ Daniela Strauß, ${ }^{1}$ Yoshihiro Yoneda, ${ }^{3}$ Jun Katahira, ${ }^{3}$ \\ Irmgard Sinning, ${ }^{1} \dagger$ Ed Hurt ${ }^{1} \dagger$
}

\begin{abstract}
Ribosomal proteins are synthesized in the cytoplasm, before nuclear import and assembly with ribosomal RNA (rRNA). Little is known about coordination of nucleocytoplasmic transport with ribosome assembly. Here, we identify a transport adaptor, symportin 1 (Syo1), that facilitates synchronized coimport of the two 5S-rRNA binding proteins Rpl5 and Rpl11. In vitro studies revealed that Syo1 concomitantly binds Rpl5-Rpl11 and furthermore recruits the import receptor Kap104. The Sy01-Rpl5-Rpl11 import complex is released from Kap104 by RanGTP and can be directly transferred onto the $5 S$ rRNA. Syo1 can shuttle back to the cytoplasm by interaction with phenylalanine-glycine nucleoporins. X-ray crystallography uncovered how the $\alpha$-solenoid symportin accommodates the Rpl5 amino terminus, normally bound to $5 S$ rRNA, in an extended groove. Symportin-mediated coimport of Rpl5-Rpl11 could ensure coordinated and stoichiometric incorporation of these proteins into pre-60S ribosomes.
\end{abstract}

$\mathrm{R}$ ibosomes perform their role in translation in the cytoplasm, but ribosome assembly occurs predominantly in a specialized nuclear compartment, the nucleolus $(1-4)$. The construction of ribosomes follows an ordered assembly of $\sim 80$ ribosomal proteins (r-proteins) and four ribosomal RNAs (rRNAs) into a small $(40 S)$ and large $(60 S)$ ribosomal subunit. This process is spatially and temporally coordinated, starting with cotranscriptional assembly of a first preribosomal particle $(90 S)$ in the nucleolus that is subsequently separated into pre- $40 S$ and pre$60 S$ ribosomes, which follow independent processing and maturation steps before export into the cytoplasm (5-8). R-proteins are synthesized in the cytoplasm and are imported into the nucleus by nuclear import receptors of the importin$\beta /$ karyopherin family $(9,10)$. These transport receptors recognize different types of nuclear localization sequences (NLSs), and hence a number of import receptors have been implicated in decoding NLSs of r-proteins in a redundant way $(9,11)$. After nuclear import and before incorporation into nascent ribosomes, r-proteins are released from the transport receptor by its interaction with RanGTP (12). At present, it is thought that each r-protein is individually transported into the nucleus by its import receptor. However, a number of r-proteins form functional clusters on the ribosomal surface or assemble

${ }^{1}$ Biochemie-Zentrum der Universität Heidelberg, Im Neuenheimer Feld 328, Heidelberg D-69120, Germany. ${ }^{2}$ Unit of Biochemistry, Department of Biology, University of Fribourg, Chemin du Musée 10, CH-1700 Fribourg, Switzerland. ${ }^{3}$ Biomolecular Networks Laboratories, Graduate School of Frontier Biosciences, Osaka University, 1-3 Yamadaoka, Suita, Osaka 565-0871, Japan.

*These authors contributed equally to this work. tTo whom correspondence should be addressed. E-mail: dieter.kressler@unifr.ch (D.K.); irmi.sinning@bzh.uni-heidelberg. de (I.S.); ed.hurt@bzh.uni-heidelberg.de (E.H.) at distinct temporal or spatial entry points during ribosome formation (13-15), thus raising the possibility of coordinated nuclear import and assembly of r-proteins.

One such pair of functionally related r-proteins is Rpl5 and Rpl11, which are close to each other on the mature $60 S$ subunit and bind to opposite sites on the $5 S$ rRNA (Fig. 1A) (13). $5 S$ rRNA transport has been extensively studied in the Xenopus oocyte system. However, this is a special case because $5 S$ rRNA is exported into the cytoplasm for storage, either with ribosomal protein L5 [5S ribonucleoprotein (RNP)] or transcription factor IIIA (7S RNP) $(16,17)$. In contrast, $5 S$ rRNA export may not occur in somatic cells, and only a few studies have addressed $5 S$ RNP biogenesis in this case. One of these investigations reported that yeast Rpl5 and Rpl11 form a ribonucleoprotein particle with the $5 S$ rRNA that could be incorporated with the aid of two biogenesis factors, Rpf2 and Rrs1, into pre-60S ribosomes (18). However, it is not known how Rpl5 and Rpl11 enter the nucleus and whether this is coordinated with their assembly onto the $5 S$ rRNA. We performed tandem affinity purification (TAP) of TAP-tagged Saccharomyces cerevisiae Rpl5 and observed coenrichment of Rpl11 and the uncharacterized yeast protein Yd1063c (Fig. 1B, lane 1). Ydl063c, which we named Syo1 (for synchronized import or briefly symportin), is present in all eukaryotes, inthermophilum and Homo sapiens. Reciprocal tagging of Syo1 with Flag-tobacco etch virus-protein $\mathrm{A}$ and subsequent affinity purification revealed copurification of Rpl5 and Rpl11, but not other r-proteins (Fig. 1B, lane 2).

Yeast two-hybrid (Y2H) assays indicated that Syo1 binds both Rpl5 and Rpl11 (Fig. 1C). To further dissect these interactions and gain highresolution structural data, we performed in vitro cluding the eukaryotic thermophile Chaetomium reconstitution studies with Syo1, Rpl5, and Rpl11 from the thermophilic fungus $C$. thermophilum (ct) (19), owing to their improved biochemical properties compared to the orthologous $S$. cerevisiae proteins. These investigations demonstrated that ctSyol can bind to either $c t$ Rpl5 or $c t$ Rpl11 (Fig. 1D). Moreover, it was possible to reconstitute the heterotrimeric $c t$ Syo1-ctRpl5-ctRpl11 complex, and size exclusion chromatography in line with static light scattering and refractive index measurements revealed a 1:1:1 stoichiometry of the three components (Fig. 1E and table S1). Likewise, we assembled a stoichiometric complex between yeast Syol and Rpl5 expressed in Escherichia coli (fig. S1B), but expression of yeast Rpl11 yielded largely insoluble protein, which hindered binding studies with Syo1. Taken together, in vivo and in vitro analyses demonstrated that Syo1 can simultaneously bind Rpl5 and Rpl11 to form a heterotrimeric Syo1-Rp15-Rp111 complex.

To gain further insight into the in vivo role of Syo1 with respect to the Rpl5-Rpl11 interaction, we returned to the yeast system. Dot spot growth analysis showed that cells with chromosomally disrupted $S Y O 1$ (syol $\Delta$ ) were viable, but exhibited reduced cell growth, especially at lower temperatures (Fig. 1F). Analysis of ribosome and polysome profiles of syol $1 \Delta$ cells showed a significant reduction of free $60 \mathrm{~S}$ subunits relative to $40 S$ subunits, and consequently, the appearance of half-mer polysomes was observed (Fig. 1G). Subsequent genetic analyses revealed a functional link between Syo1 and Rp15, as evidenced by (i) synthetic lethality between syol $\Delta$ and $r p l 5$ alleles, (ii) high-copy suppression of the cold-sensitive syol $\triangle$ phenotype by RPL5, and (iii) a complete rescue of the slow-growth or lethal phenotypes of distinct rpl5 mutant alleles (e.g., rpl5L104S) by Syo1 overexpression (Fig. $1 \mathrm{H}$ and fig. S2). Together, these data point to a role for Syol in ribosome synthesis, possibly by facilitating assembly of Rp15-Rpl11 onto the pre- $60 \mathrm{~S}$ subunit.

To obtain insight into the function of Syo1 at the atomic level, we determined the crystal structure of $c t$ Syo1 at $2.1 \AA$ resolution by singleanomalous dispersion (SAD) (table S2). Syo1 forms an all $\alpha$-helical elongated superhelix or $\alpha$-solenoid (Fig. 2A), which is typical for nuclear transport receptors $(20,21)$. Whereas importin- $\alpha$, the import adaptor for classical NLSs, exclusively contains ARM repeats, importin- $\beta$ transport receptors are exclusively composed of HEAT repeats. Syo1 is an unusual chimera of four complete ARM repeats (residues 65 to 260) followed by six HEAT repeats (residues 274 to 675), whose inner surfaces form an extensive groove (Fig. 2, A and B). Karyopherins contain binding sites for NLSs and phenylalanine-glycine (FG) repeats of nucleoporins on their inner and outer $\alpha$-solenoid surfaces, respectively (22). By analogy, Syo1 may act as a nuclear import receptor or adaptor by binding to a linear motif of Rpl5 or Rpl11 and/or interacting with FG repeats.

Rpl5 is composed of a globular domain flanked by $\mathrm{N}$ - and C-terminal extensions that clamp the 
$5 S$ rRNA within the $60 S$ subunit (13) (see also Fig. 1A). $\mathrm{Y} 2 \mathrm{H}$ analyses and in vitro binding assays revealed that the N-terminal 41 amino acids of Rp15 (L5-N) are required and sufficient for a robust interaction with Syol (fig. S1). To gain structural insight into the Syo1-Rpl5 interaction, we determined the crystal structure of ctSyo1 in complex with $c t \mathrm{~L} 5-\mathrm{N}$ at $2.95 \AA$ resolution by molecular replacement using the $c t$ Syol structure as the search model (Fig. 2, B and D, and table S2). The side chains and main-chain carbonyls were well defined in the electron density map, and residues 2 to 20 of $c t R p 15$ could be unambiguously traced (Fig. 2, C and D). L5-N forms an elongated peptide chain with a short helical segment $\left(\mathrm{Tyr}^{12}\right.$ to $\mathrm{Phe}^{16}$ ), which binds into the extended groove formed by $c t$ Syol and primarily interacts with residues of HEAT repeats 1 to 4 of ctSyol (binding interface: $980 \AA^{2}$; for a more detailed description, see fig. S3). Therefore, Rpl5 binding to Syo1 results in extensive shielding of an Rp15 region involved in $5 S$ rRNA binding. Moreover, L5-N binding induces a slight conformational rearrangement of the ARM and HEAT domains of Syo1 (fig. S4). L5-N binding to Syo1 seems to slightly "open" the $\alpha$-solenoid structure of Syo1, which could be of functional relevance but may also reflect a crystal-packing artifact. Taken together, the mode of Rpl5 recognition by Syo1 is reminiscent of the interaction between linear motifs (e.g., from
Nup2) and the $\alpha$-solenoid surface of importin- $\alpha$ $(23,24)$ (fig. S5).

In the case of Rpl11, we could not restrict the primary sequence into a linear motif that efficiently binds to Syo1. This implies that the interaction between Rpll1 and Syol could be more complex, possibly involving part of the Rpl11 fold (see Fig. 1A for Rpl11 structure). To determine the Rpl11 binding surface on Syo1, we performed ${ }^{1} \mathrm{H} /{ }^{2} \mathrm{H}$ exchange mass spectrometry (HX-MS) labeling experiments. Specifically, free $c t S y o 1$ and the $c t$ Syo1-ctRpl11 heterodimer were, after completion of the HX labeling, digested with pepsin, and the resulting peptide fragments were analyzed by electrospray ionization-mass spectrometry. Only
A

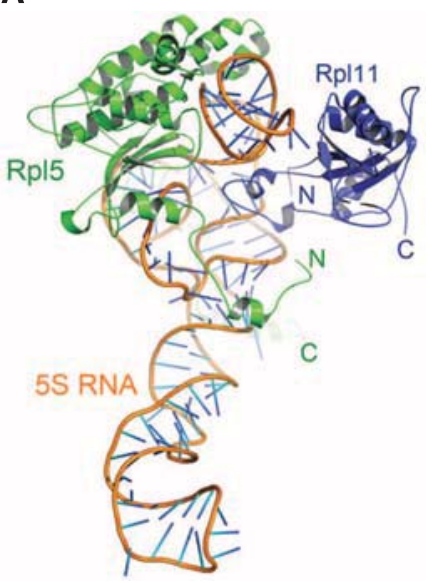

B

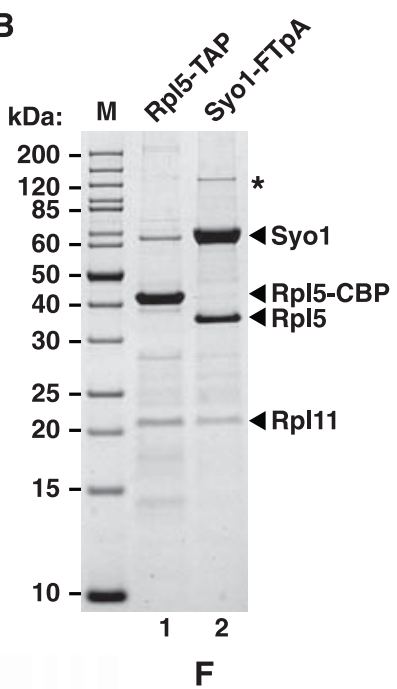

C

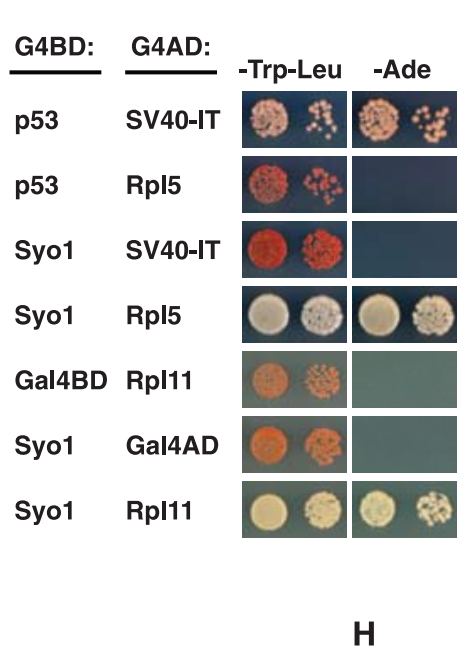

D

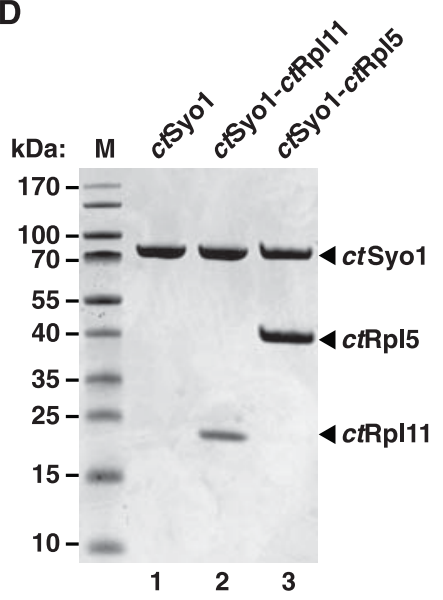

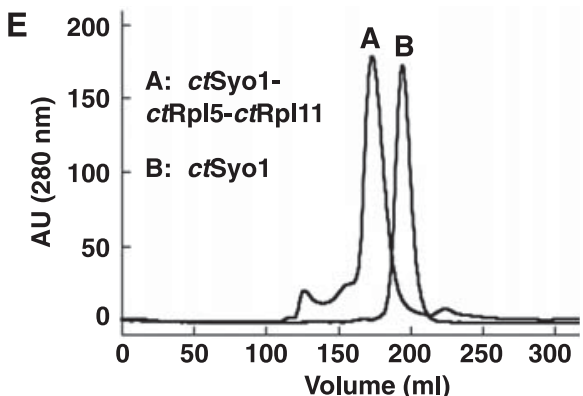

kDa: peak fractions trimeric complex (A)

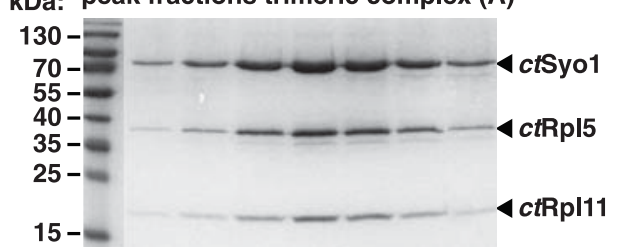

Fig. 1. Syo1 forms a trimeric complex with Rpl5 and Rpl11. (A) Structure of the $5 S$ RNP (5S rRNA-Rpl5-Rpl11) as observed in the S. cerevisiae 805 ribosome (PDB 3U5D and 3U5E) (13). The 5S rRNA is shown in orange, Rpl5 in green (residues 2 to 297), and Rpl11 in blue (residues 6 to 174). N and C termini are labeled in the respective colors. (B) Identification of Syo1 as an Rpl5 binding protein. Tandem-affinity purification of TAP-tagged Rpl5 (lane 1) and FTpA-tagged Syo1 (lane 2) from yeast cell lysates. Final eluates were analyzed by SDS-PAGE (polyacrylamide gel electrophoresis) and Coomassie staining. The indicated proteins were identified by mass spectrometry. Asterisk indicates the common contaminant Tif4631. M, molecular weight standard. (C) Y2H interaction between Syo1 and Rpl5 or Rpl11. (D) In vitro binding assay between $c$ Syo1 and ctRpl5 or ctRpl11. Proteins were (co-)expressed in E. coli,
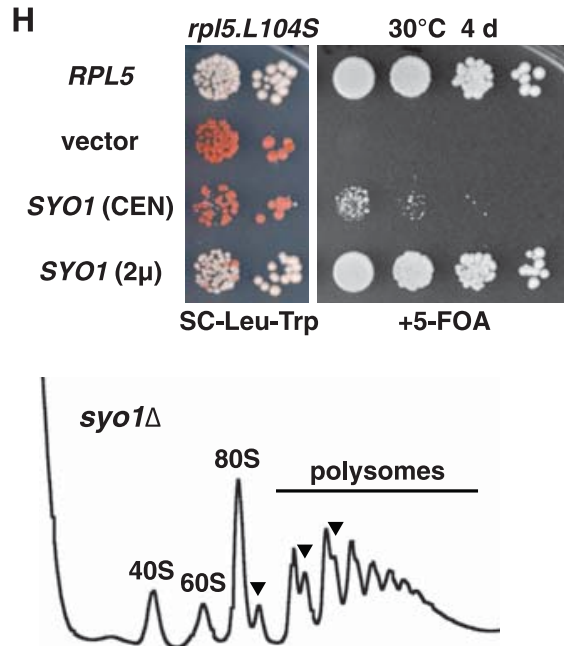

purified via Ni-affinity purification and revealed by SDS-PAGE and Coomassie staining. Lanes: 1, ctSyo1-(His) 6 ; 2, ctSyo1-(His) $6 /$ ctRpl11; 3, ctSyo1/ctRpl5-(His) $6 ;$ M, molecular weight standard. (E) Size exclusion chromatography of reconstituted ctSyo1/ctRpl5-(His) $6 / c t R p l 11$ heterotrimer (chromatogram A) and ctSyo1 (chromatogram B). The peak fractions of chromatogram A were analyzed by SDS-PAGE and Coomassie staining. (F) The syo1 $\Delta$ null mutant displays a slow-growth phenotype at low temperatures. Growth analysis of wild-type (SYO1) and isogenic syo1 $\triangle($ syo1 $\Delta$ ) deletion mutant. (G) Syo1 is required for efficient synthesis of 605 ribosomal subunits. Polysome profiles of wild-type (SYO1) and syo1 $\Delta$ (syo1 $\Delta$ ) cells grown at $23^{\circ} \mathrm{C}$. Free $40 \mathrm{~S}$ and $60 \mathrm{~S}$ subunits, $80 \mathrm{~S}$ ribosomes, and polysomes are indicated; arrowheads indicate half-mer polysomes. (H) Overexpression of Syo1 rescues the lethal phenotype of the $r p l 5 L 104 S$ allele. 
the very $\mathrm{C}$-terminal part of the $c t$ Syol disordered loop (residues 395 to 408) showed a decrease in deuterium incorporation upon $c t$ Rpl11 binding, indicating shielding of this ctSyol region (fig. S6A). Consistent with these data, deletion of residues 393 to 397 in the disordered loop abolished binding to $c t$ Rpl11 (fig. S6, B and C). Moreover, $\mathrm{Y} 2 \mathrm{H}$ assays indicated that the ARM domain of Syo1 contributes to Rpl11 binding (fig. S7). From these results, we conclude that the disordered loop of Syo1 in conjunction with the ARM domain provides the interaction site for Rpl11 (for a model, see fig. S8).

To act as import receptor, Syo1 must pass through the hydrophobic FG-meshwork of the nuclear pore complex (NPC) transport channel. Shuttling karyopherin transport receptors do so by binding with low (micromolar) affinity to FG repeats of nucleoporins $(12,25)$. We found that Syo1 and ctSyo1 bind to FG-rich repeats of distinct nucleoporins with a binding constant in the low micromolar range (Fig. 3, A and B, and fig. S9, A and B). Moreover, Syo1 can translocate into the nucleus on its own in the absence of cytosol, which provides karyopherin transport receptors and RanGTP, when tested in an in vitro nuclear import assay with permeabilized $\mathrm{HeLa}$ cells $(26,27)$ (Fig. 3C and fig. S9, C and D). In agreement with translocation across the NPC channel, Syo1 did not show nuclear accumulation when permeabilized HeLa cells were incubated with wheat germ agglutinin (WGA), a specific inhibitor of nucleocytoplasmic transport that sterically blocks the FG-repeat meshwork of the NPC transport channel (Fig. 3E and fig. S9, $\mathrm{C}$ and $\mathrm{D})$. Thus, Syol can directly pass through the NPC by low-affinity interaction with FG repeats of NPC-channel nucleoporins.

However, and in contrast to $c t$ Syol alone, the reconstituted ctSyo1-ctRpl11, ctSyo1-ctRpl5, and $c t$ Syo1-ctRp15-ctRpl11 complexes were inefficiently imported into the nucleus in the absence of cytosol and energy (Fig. 3C and fig. S10B). Consistent with this observation, the $c t$ Syo1-ctRpl11 and $c t$ Syo1-ctRp15-ctRp111 complexes, and to a lesser extent the $c t$ Syo1-ctRpl5 complex, exhibited reduced binding to $\mathrm{FG}$ repeats (Fig. 3B and fig. S10C), suggesting that recruitment of Rp15/Rp111 masks or alters the FG-binding site(s) on Syo1. Notably, nuclear import of the ctSyo1$c t$ Rpl5-ctRpl11 complex was markedly stimulated by addition of cytosol and energy (Fig. 3C), but did not occur at $4^{\circ} \mathrm{C}$ or in the presence of WGA or the guanosine triphosphatase (GTPase)-deficient Ran(Q69L) mutant (28) (Fig. 3, D and E). Thus, we conclude that a RanGTP-dependent importin- $\beta$ transport receptor mediates nuclear import of the Syo1-Rp15-Rpl11 complex. Moreover, Syo1, released from Rpl5 and Rpl11 after nuclear import, could shuttle back to the cytoplasm on its own. In support of this possibility, Syol alone traverses the NPC in both directions in permeabilized HeLa cells (Fig. 3 and fig. S9), and Syo1GFP (green fluorescent protein) is located both in the nucleus and cytoplasm in yeast cells (fig. S12, C and D).

To identify the import receptor of the Syo1Rpl5-Rpl11 complex, we took advantage of the observation that low amounts of Kap104 were sometimes coenriched when Syo1 was affinitypurified from yeast (fig. S11A). Kap104 is a member of the conserved karyopherin- $\beta 2 /$ transportin receptor subfamily (9), which recognizes a nonclassical NLS of the hydrophobic or basic prolinetyrosine (PY)-NLS type with the C-terminal consensus signature $\mathrm{R} / \mathrm{K} / \mathrm{H}-\mathrm{X}_{2-5}-\mathrm{P}-\mathrm{Y} / \mathrm{L}(29,30)$ (fig. S11B). Notably, Syo1 contains a conserved putative basic PY-NLS at its N terminus. In vitro binding assays revealed that this PY-NLS is necessary and sufficient for the interaction of Syol with Kap104 (fig. S11C and fig. S12A). Moreover, the PY-NLS can target an attached GFP reporter into the nucleus in vivo and is required for the in vivo function of Syo1 and nuclear

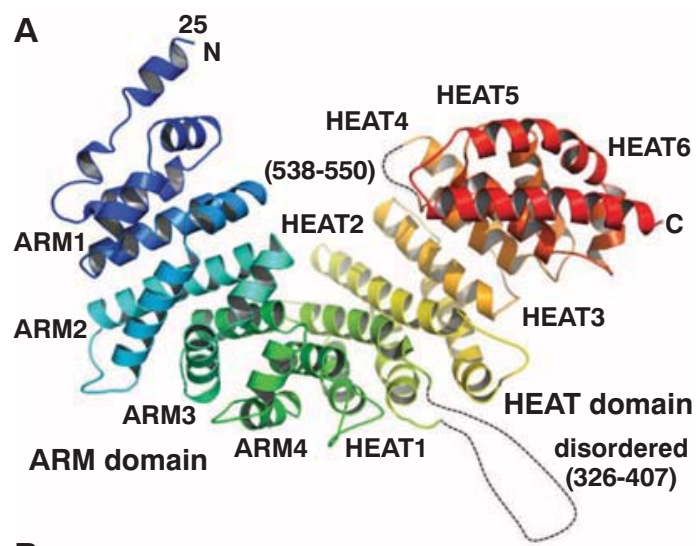

B

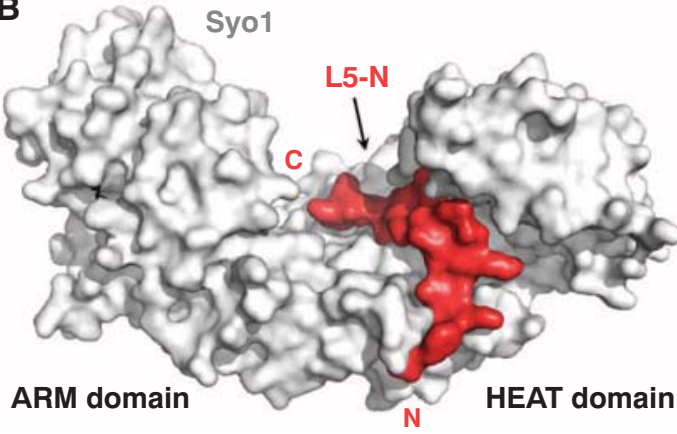

Fig. 2. Syo1 is structurally related to importins and binds the N-terminal residues of Rpl5 in an extended groove. (A) Crystal structure of ctSyo1 (residues 25 to 675). Cartoon representation showing that $c t$ Syo1 is an extended $\alpha$-solenoid, which consists of four complete ARM repeats (residues 65 to 260) followed by six HEAT repeats (residues 274 to 675 ). The ARM and HEAT repeats are indicated and "rainbow" colored from the $\mathrm{N}$ to the $\mathrm{C}$ terminus. Dashed lines indicate disordered regions. (B) Surface representation of the ctSyo1/ctL5-N complex. Residues 2 to 20 of $c t R p l 5$ (L5-N, red) are accommo-

C
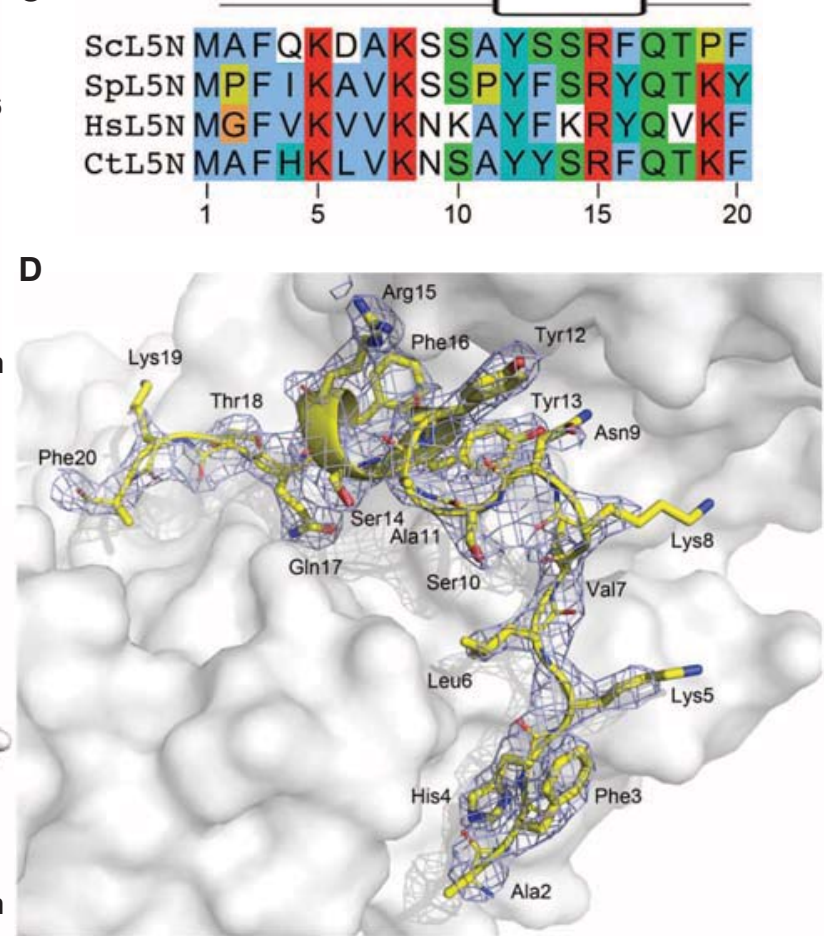

dated in an extensive groove of ctSyo1 (gray). (C) Multiple sequence alignment of residues 2 to 20 of L5-N from S. cerevisiae (Sc), Schizosaccharomyces pombe (Sp), Homo sapiens ( $\mathrm{Hs})$, and $C$. thermophilum (Ct). Single-letter abbreviations for the amino acid residues are as follows: $A, A$ ala; D, Asp; F, Phe; G, Gly; H, His; I, Ile; K, Lys; L, Leu; M, Met; N, Asn; P, Pro; Q, Gln; R, Arg; S, Ser; T, Thr; V, Val; and Y, Tyr. (D) Enlargement of the ctL5-N/ctSyo1 interaction. The $2 F_{\text {obs }}-F_{\text {calc }}$ electron density of $c t \mathrm{~L} 5-\mathrm{N}$ at $1.3 \sigma$ after final refinement is shown. 
localization of Syo1-GFP (fig. S11D and fig. $\mathrm{S} 12$, B and C). Consistent with the proposal that Kap104 is the predominant import receptor of
Syo1, we could not observe nuclear accumulation of Syo1-GFP in temperature-sensitive kap104-16 mutant cells (31) (fig. S12D).
To test the model of Kap104 as the principal transport receptor for a trimeric import complex comprising Syo1-Rp15-Rp111, we reconstituted the
A

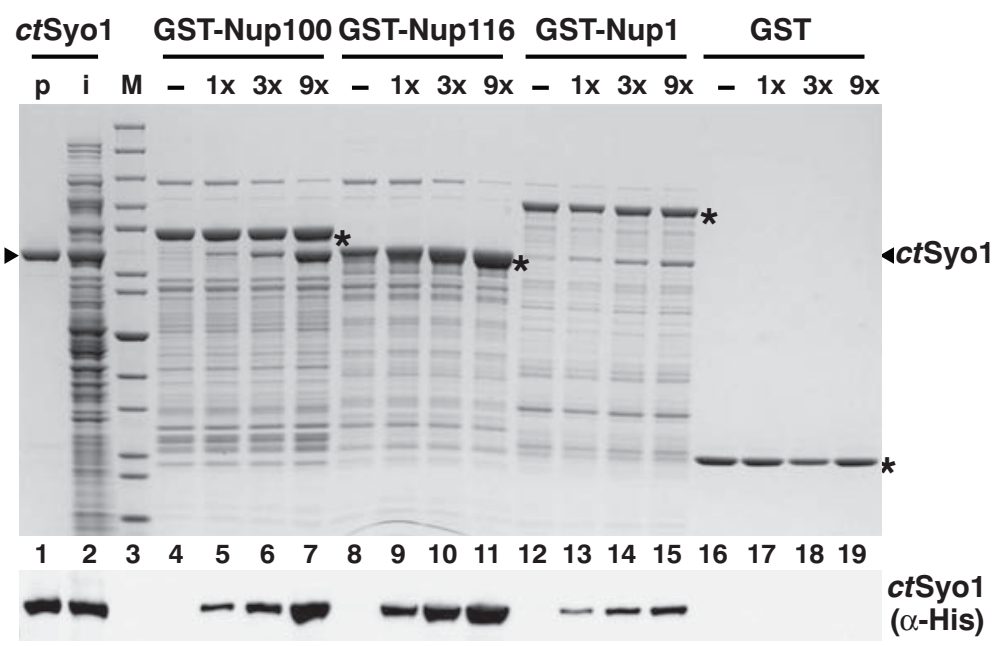

B

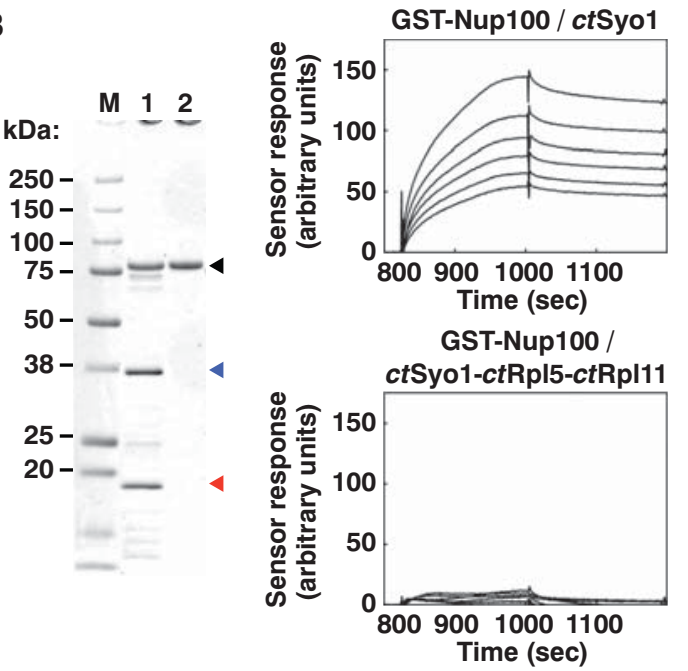

C

ctSyo1-Flag ctSyo1-Flag ctRpl5-ctRpl11

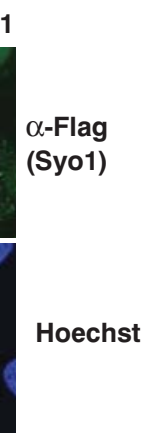

\section{D}

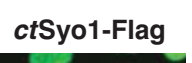

ctSyo1-Flag

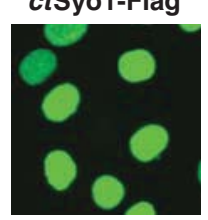

ctRpl5-ctRpl11
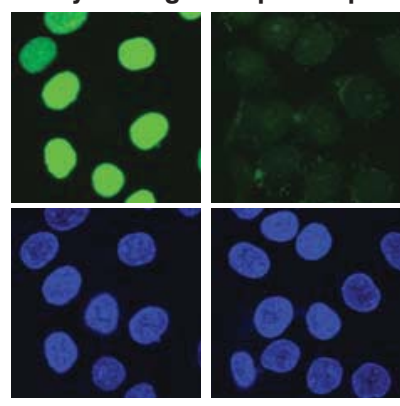

$\alpha$-Flag

(Syo1)

buffer
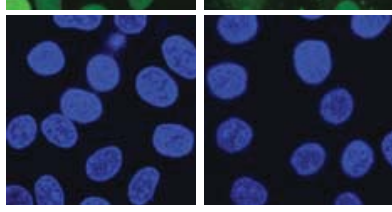

ctSyo1-Flag

ctSyo1-Flag ctRpl5-ctRpl11
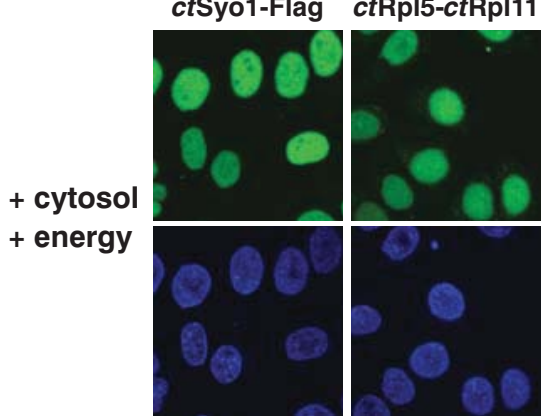

$\alpha$-Flag

(Syo1)

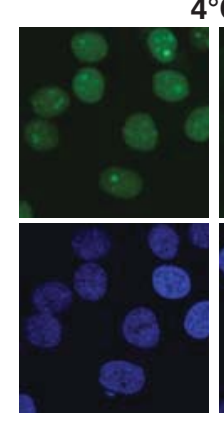

$4^{\circ} \mathrm{C}$

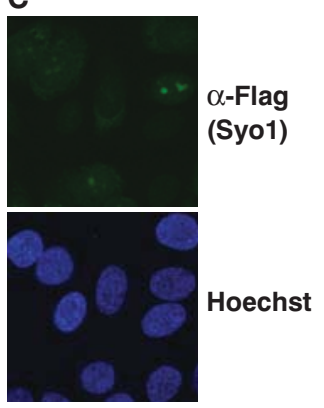

E

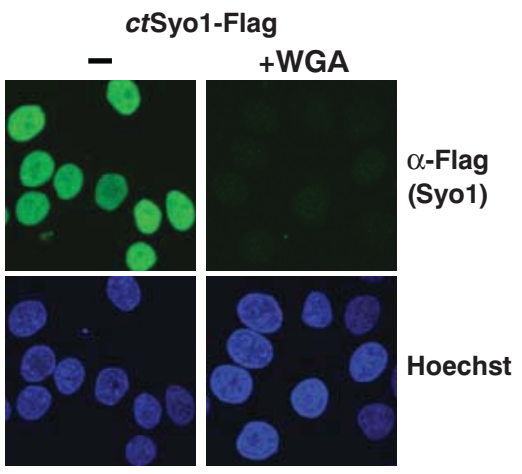

ctSyo1-Flag

ctRpl5-ctRpl11
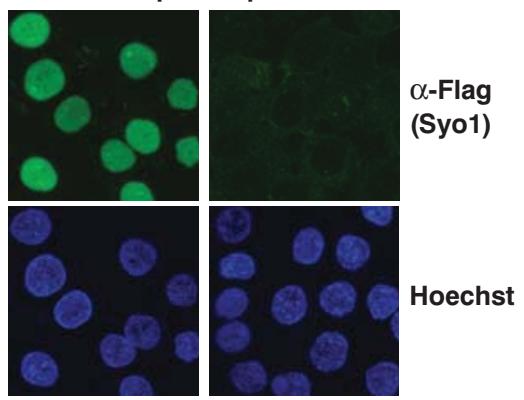

Fig. 3. The Syo1-Rpl5-Rpl11 complex is actively imported into the nucleus. (A and B) ctSyo1 interacts with FG repeats of nucleoporins. (A) Binding of recombinant ctSyo1 to immobilized FG-repeat domains of nucleoporins (GSTNup) in the presence of competitor E. coli lysate. Lane 1, purified ctSyo1 (p); lane 2, ctSyo1 mixed with $E$. coli lysate (input, i); lane 3, molecular weight standard (M), same as in Fig. 1B; lanes 4 to 19, proteins bound to the indicated glutathione S-transferase (GST)-Nups or the GST control in the absence $(-)$ or presence of increasing amounts of $c t$ Syo1 $(1 x, 3 x, 9 x)$. Bound proteins were analyzed by SDS-PAGE and Coomassie staining (upper panel) or Western analysis (lower panel) with anti-penta-His antibody (ctSyo1). (B) BIAcore analysis of the interaction between the FG-repeat domain of Nup100 and ctSyo1 or the trimeric ctSyo1-ctRpl5-ctRpl11 complex. Protein input for BIAcore measurement (left panel); ctSyo1-Flag-(His) 6 (lane 2) and ctSy01-Flag/ctRpl5/ctRpl11-(His) 6 (lane 1 ) were purified by nickel-ion affinity chromatography and analyzed by SDS-PAGE and Coomassie staining. Black, blue, and red arrowheads indicate

ctSyo1-Flag/ctSyo1-Flag-(His) ${ }_{6}, c t R p l 5$, and $c t$ Rpl11-(His) $)_{6}$, respectively. $M$ molecular weight standard. GST-Nup100 was immobilized and different concentrations $\left(0.09\right.$ to $0.48 \mu \mathrm{M}$ ) of purified $c t$ Syo1-Flag-(His) ${ }_{6}$ (upper right panel) or ctSyo1-Flag/ctRpl5/ctRpl11-(His) 6 (lower right panel) were injected over the sensor chip, and sensor responses (arbitrary units) were recorded. (C to E) In vitro nuclear import assays. HeLa cells were permeabilized by digitonin and incubated with purified ctSyo1 or ctSyo1-ctRpl5-ctRpl11 complex in transport buffer $[(C)$, upper panel], transport buffer containing either cytosol/energy (guanosine triphosphate/adenosine triphosphate) at $30^{\circ} \mathrm{C}[(\mathrm{C})$, lower panel] or $4^{\circ} \mathrm{C}[(D)$, lower panel], or cytosol/energy/Ran(Q69L) [(D), upper panel]. Permeabilized HeLa cells were first treated with transport buffer containing cytosol/energy in the absence or presence of WGA before performing the import reactions (E). Nuclear translocation of $c t$ Syo1 or the trimeric complex was revealed by indirect immunofluorescence against Flag-tagged ctSyo1. Nuclei were revealed by staining DNA with Hoechst. 
interaction of Kap104 with this complex using recombinant proteins derived from either $C$. thermophilum or yeast (Fig. 4A and fig. S12E). Size-exclusion chromatography revealed formation of a stable $c t$ Kap104-ctSyo1-ctRpl5-ctRpl11 import complex (Fig. 4A) with a 1:1:1:1 stoichiometry of the four components, as determined by static light scattering and refractive index measurements (table S1). Addition of RanGTP was sufficient to release the trimeric $c t$ Syo1-ctRpl5$c t$ Rpl11 cargo complex from $c$ Kap104 (Fig. 4B). These findings indicate that Syol can serve as the import adaptor for Rp15-Rpl11 with Kap104 as the cognate import receptor for the hetero- trimer. Given that Syo1 is not an essential protein, it is apparent that alternative import routes for Rpl5 and Rpl11 must exist, which could involve other transport receptors or diffusion across the NPC.

After nuclear transport and RanGTP-mediated release of Syo1-Rp15-Rpl11 from Kap104, the complex could be transferred onto the newly synthesized $5 S$ rRNA in the nucleus. To test the possibility of a direct transfer in vitro, we produced $C$. thermophilum $5 S$ rRNA by in vitro transcription and added it to the preformed ctSyo1-ctRpl5-ctRpl11 complex (Fig. 4, C and D). This binding assay revealed that $5 S$ rRNA was efficiently bound to $c t$ Syo1-ctRpl5-ctRpl11, as evidenced by a strict coelution of heterotrimer and rRNA on the gel filtration column (Fig. 4D). In contrast, $5 S$ rRNA alone eluted in later fractions from this column. Thus, $5 S$ rRNA can directly bind to the Syo1-Rpl5-Rpl11 complex. Removal of Syol from the $5 S$ rRNP likely occurs at a subsequent maturation step, possibly during incorporation of the $5 S$ RNP into preribosomal particles, which may require also other factors, such as Rpf2 and Rrs1 (18).

Our study has uncovered a mechanism that allows simultaneous nuclear import of more than one cargo, coupling nucleocytoplasmic transport
A

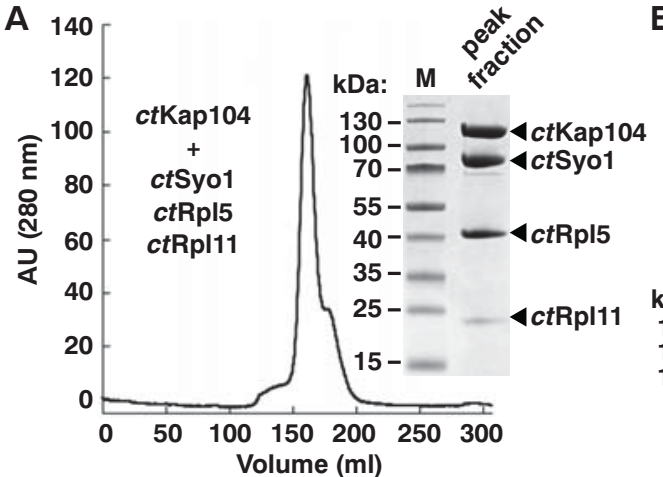

C

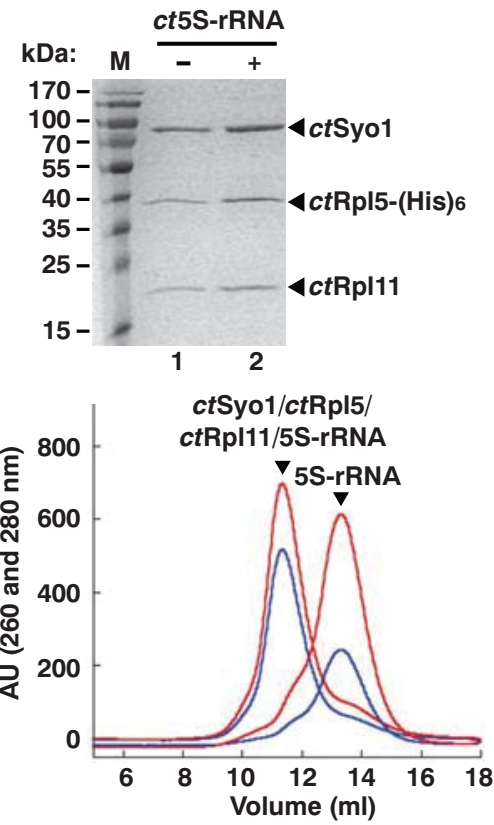

B

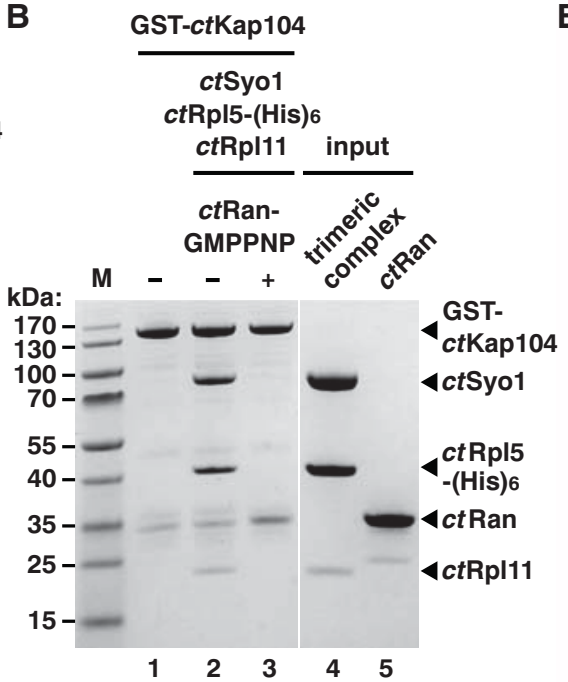

E
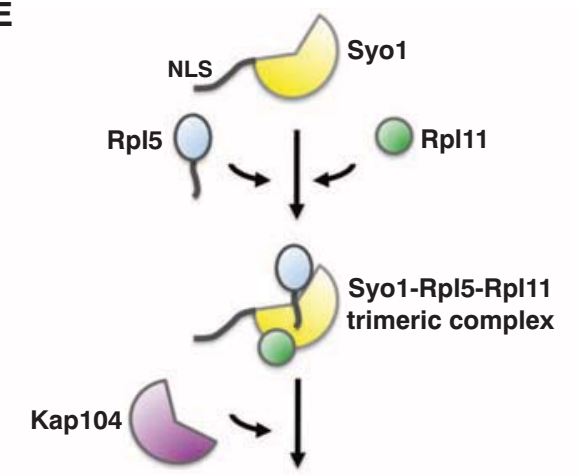

M Coomassie-stained SDS-PAGE
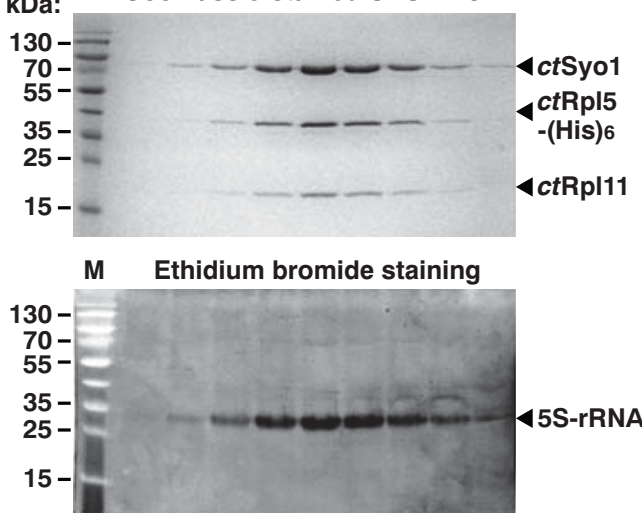

Kap104Syo1-Rpl5-Rpl11 import complex

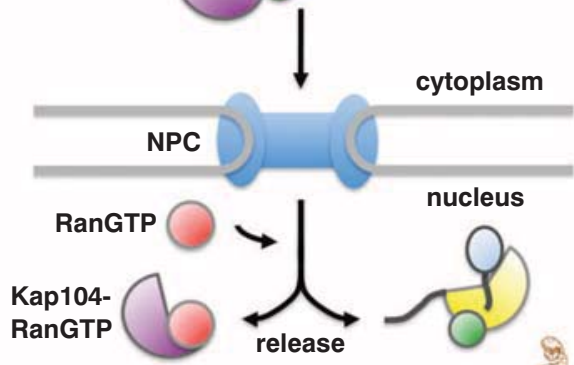

RanGTP

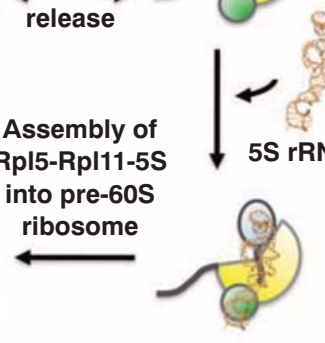

Fig. 4. The Syo1-Rpl5-Rpl11 complex is imported into the nucleus via Kap104 and binds to the 5S rRNA. (A) Size exclusion chromatography of the reconstituted ctKap104-ctSyo1-ctRpl5-ctRpl11 heterotetrameric import complex. The peak fraction was analyzed by SDS-PAGE and Coomassie staining. (B) RanGMPPNP promotes release of the ctSyo1-ctRpl5-ctRpl11 complex from ctKap104. The trimeric ctSyo1-ctRpl5-ctRpl11 complex (lane 4) was bound to immobilized GST-ctKap104 (lane 1) and incubated in the absence (lane 2) or presence (lane 3) of RanGMPPNP (lane 5). Input and bound proteins were revealed by SDS-PAGE and Coomassie staining. $M$, molecular weight standard. (C) Coomassie-stained SDS-PAGE of an in vitro binding assay of ctSyo1$c t$ Rpl5-ctRpl11 in the absence or presence of $c t 5 S$ rRNA. The presence of $5 S$ rRNA did not lead to disassembly of the $c t$ Syo1-ctRpl5-ctRpl11 complex. (D) The $c t 5 S$ rRNA and $c t$ Syo1-ctRpl5-ctRpl11 form a stable complex on size ex- clusion chromatography. Size exclusion chromatogram for free $c t 5 S$ rRNA and the ct5S rRNA-ctSyo1-ctRpl5-ctRpl11 complex at two different wavelengths [i.e., $260 \mathrm{~nm}$ (red) and $280 \mathrm{~nm}$ (blue), upper panel]. SDS-PAGE of the peak fractions stained with Coomassie (middle panel) and ethidium bromide (lower panel) to detect the proteins and the $5 S$ rRNA, respectively, present in the $c t 5 S$ rRNA-ctSyo1-ctRpl5-ctRpl11 complex. (E) The transport adaptor Syo1 synchronizes nuclear import of Rpl5 and Rpl11. Syo1 simultaneously interacts with Rpl5 and Rpl11 in the cytoplasm, thus enabling formation of a tetrameric Kap104-Syo1-Rpl5-Rpl11 import complex. RanGTP binding to Kap104 in the nucleus promotes release of the trimeric Syo1-Rpl5-Rpl11 complex, which recruits the $5 S$ rRNA. Rpl5, Rpl11, and the $5 S$ rRNA are then incorporated with the aid of assembly factors (not shown) into pre-60S ribosomes. See text for details. 
to stoichiometric assembly of proteins into macromolecular machines. Key to this mechanism is a nuclear import adaptor, Syo1, that specifically recruits the two functionally and topologically linked r-proteins Rpl5 and Rpl11. It guarantees that this cargo pair remains bound together from the time of synthesis in the cytoplasm until delivery to the nascent $5 S$ rRNA in the nucleus (see Fig. 4E). In the broader sense, synchronous nuclear transport of topologically linked and/or functionally related cargo may represent a general strategy to streamline downstream nuclear processes that depend on temporally or spatially controlled assembly steps.

\section{References and Notes}

1. F. M. Boisvert, S. van Koningsbruggen, ]. Navascués, A. I. Lamond, Nat. Rev. Mol. Cell Biol. 8, 574 (2007).

2. D. L. Lafontaine, D. Tollervey, Nat. Rev. Mol. Cell Biol. 2, 514 (2001).

3. T. Pederson, R. Y. Tsai, J. Cell Biol. 184, 771 (2009).

4. J. R. Warner, Trends Biochem. Sci. 24, 437 (1999).

5. A. K. Henras et al., Cell. Mol. Life Sci. 65, 2334 (2008).

6. D. Kressler, E. Hurt, J. Bassler, Biochim. Biophys. Acta 1803, 673 (2010).

7. J. P. Staley, J. L. Woolford Jr., Curr. Opin. Cell Biol. 21, 109 (2009).

8. H. Tschochner, E. Hurt, Trends Cell Biol. 13, 255 (2003).

9. Y. M. Chook, K. E. Süel, Biochim. Biophys. Acta 1813 1593 (2011)

10. M. P. Rout, G. Blobel, ]. D. Aitchison, Cell 89, 715 (1997).

11. D. Görlich, U. Kutay, Annu. Rev. Cell Dev. Biol. 15, 607 (1999).
12. M. Stewart, Nat. Rev. Mol. Cell Biol. 8, 195 (2007).

13. A. Ben-Shem et al., Science 334, 1524 (2011).

14. S. Ferreira-Cerca et al., Mol. Cell 28, 446 (2007).

15. G. Pöll et al., PLOS ONE 4, e8249 (2009).

16. M. Ciganda, N. Williams, Wiley Interdiscip. Rev. RNA 2 523 (2011).

17. M. Claussen, F. Rudt, T. Pieler, J. Biol. Chem. 274, 33951 (1999).

18. ]. Zhang et al., Genes Dev. 21, 2580 (2007)

19. S. Amlacher et al., Cell 146, 277 (2011).

20. M. A. Andrade, C. Petosa, S. I. O'Donoghue, C. W. Müller P. Bork, J. Mol. Biol. 309, 1 (2001).

21. D. Xu, A. Farmer, Y. M. Chook, Curr. Opin. Struct. Biol. 20, 782 (2010).

22. K. E. Süel, A. E. Cansizoglu, Y. M. Chook, Methods 39, 342 (2006).

23. M. Marfori et al., Biochim. Biophys. Acta 1813, 1562 (2011).

24. Y. Matsuura, M. Stewart, EMBO ]. 24, 3681 (2005).

25. R. Bayliss, T. Littlewood, L. A. Strawn, S. R. Wente, M. Stewart, J. Biol. Chem. 277, 50597 (2002)

26. S. A. Adam, R. S. Marr, L. Gerace, J. Cell Biol. 111, 807 (1990).

27. B. Bradatsch et al., Mol. Cell 27, 767 (2007).

28. F. R. Bischoff, C. Klebe, ]. Kretschmer, A. Wittinghofer, H. Ponstingl, Proc. Natl. Acad. Sci. U.S.A. 91, 2587 (1994).

29. B. J. Lee et al., Cell 126, 543 (2006)

30. K. E. Süel, H. Gu, Y. M. Chook, PLoS Biol. 6, e137 (2008)

31. J. D. Aitchison, G. Blobel, M. P. Rout, Science 274, 624 (1996)

Acknowledgments: We thank A. Hendricks and C. Déforel for technical assistance, J. Kopp and C. Siegmann from the BZH Cluster of Excellence:CellNetworks crystallization platform, J. Lechner and his team for mass spectrometry, T. Ruppert and M. Mayer (Zentrum für Molekulare Biologie Heidelberg) for access to the core facility for mass spectrometry for the HX-MS experiments, ]. Woolford for sharing published $r p / 5$ mutant plasmids, and L. Dimitrova (Hurt laboratory) for providing the full-length ctKAP104 clone. Data collection was performed at the European Synchrotron Radiation Facility, Grenoble. This work was funded by the German Research Council (Hu363/9-4 to E.H. and SFB 638 to I.S.), the Swiss National Science Foundation (PPOOP3_123341 to D.K.), and the Japan Society for the Promotion of Science (no. 21570195 and no. 21247032 to J.K. and Y.Y., respectively). I.S. and E.H. are investigators of the Cluster of Excellence:CellNetworks, and G.B. is a fellow of the Peter and Traudl Engelhorn Foundation. D.K., G.B., I.S., and E.H. conceived the experiments. D.K., G.B. J.K., I.S., and E.H. analyzed the data. D.K., G.B., I.S., and E.H. wrote the paper. D.K. and D.S. constructed yeast strains and carried out all yeast genetic experiments. D.K., G.B., and S.A constructed plasmids. D.K. carried out tandem-affinity purifications of yeast proteins. G.B. and D.K. performed binding assays. G.B. determined crystal structures. G.S. carried out the HX-MS experiments. D.P. and D.S. performed fluorescence microscopy of live yeast cells. B.B. performed in vitro FG-repeat binding assays. Y.O. performed in vitro import assays with permeabilized HeLa cells under the supervision of J.K. in the laboratory of Y.Y. J.K. measured binding constants by BIAcore. D.K. and G.B. contributed equally to this study. All authors commented on the manuscript. Atomic coordinates and structure factors for the reported crystal structures have been deposited with the Protein Data Bank (PDB) under accession codes 4GMNO (ctSyo1) and 4GMN (ctSyo1/ctL5-N). The authors declare no competing financial interests. Correspondence and requests for materials should be addressed to D.K. (dieter. kressler@unifr.ch), I.S. (irmi.sinning@bzh.uni-heidelberg.de), or E.H. (ed.hurt@bzh.uni-heidelberg.de).

\section{Supplementary Materials}

Materials and Methods

Figs. $\mathrm{S} 1$ to $\mathrm{S} 12$

Tables $\mathrm{S} 1$ to $\mathrm{S} 5$

References (32-50) 